\title{
Visual recognition memory in reflective and impulsive children*
}

\author{
ALEXANDER W. SIEGEL $\dagger$, JOANNE M. BABICH, and KATHLEEN C. KIRASIC \\ University of Pittsburgh, Pittsburgh, Pennsylvania 15260
}

\begin{abstract}
Twenty-nine reflective and 29 impulsive fifth-grade boys were tested in a forced-choice visual recognition memory task. In three of the experimental conditions (1FD, 2FD, 4FD) the number of visual feature differences between the correct and incorrect test stimuli was 1,2 , or 4 , and correct response could not be based on the name of the stimulus; in the fourth condition (DO) the correct and incorrect test stimuli had different names. As predicted, performance on DO and 4FD was equivalent and was superior to that on 1FD and 2FD. Although reflective Ss made more correct responses than impulsive $\mathrm{Ss}_{\mathrm{s}}$ in all four conditions, only the performance difference in Condition $1 \mathrm{FD}$ was significant. Mean correct response latencies mirrored the correct response data. These results were consistent with the Selfridge-Neisser feature-testing model of recognition memory, and it was argued that the primary underlying basis for the dimension of reflection-impulsivity was that reflective Ss tend to engage in a more detailed visual feature analysis of stimulus arrays. Strong inferential evidence was provided that visual feature analysis independent of verbal labeling was responsible for successful recognition performance in these Ss.
\end{abstract}

The dimension of reflection-impulsivity (R-I) is purported to be a reliable and useful dimension along which to conceptualize individual differences in cognitive style. A child's relative position on this dimension is typically determined by his performance on the Matching Familiar Figures Test (MFF) (Kagan \& Kogan, 1970; Kagan, Rosman, Day, Albert, \& Phillips, 1964). In the MFF, $S$ is shown a standard stimulus and is then asked to choose the one of several strikingly similar variants that exactly matches the standard. Children who respond slowly and make relatively few errors are classified as "reflective," while children who respond quickly and make many errors are classified as "impulsive."

On the assumption that performance differences between reflective and impulsive child ren reflect a broad and pervasive dimension of individual difference approaches to problems involving high response uncertainty (Kagan et al, 1964), much research has been devoted to demonstrating that reflective and impulsive children differ in their performance on a variety of tasks. Performance of reflective children has been found to be superior to that of impulsive children on tasks of reading (Kagan, 1965), serial learning (Kagan, 1966), inductive reasoning (Kagan, Pearson, \& Welch, 1966), and hypothesis testing (Nuessle, 1972).

\footnotetext{
*The research was supported by the Learning Research and Development Center, and in part by the National Institute of Education (NIE), United States Department of Health, Education, and Welfare. The opinions expressed do not necessarily reflect the position or policy of NIE, and no of ficial endorsement should be inferred. The authors would like to acknowledge the cooperation of Sisters Eva Marie, Kathleen Marie, and Mary Jo, principals of Holy Family, St. Lawrence O'Toole, and St. Kieren's Schools, respectively.

tRequests for reprints should be sent to Alex ander W. Siegel, Department of Psychology, University of Pittsburgh, Pittsburgh, Pennsylvania 15260.
}

Zelniker, Jeffrey, Ault, and Parsons (1972) recorded eye fixations on the MFF and proposed that impulsive children have less adequate strategies for searching the stimulus complex. Odom, McIntyre, and Neale (1971) found that on a perceptual learning task, reflective children perceived and evaluated information based on the feature differences of stimulus arrays; the information processed by the impulsive children could not be identified. Thus, it is possible that reflective-impulsive performance differences reflect differences in a specific visual process rather than in broad "cognitive" predispositions. Although Zelniker et al (1972) suggest that reflective and impulsive children differ in their perceptual approach to the task and Drake (1970) and Odom et al (1971) suggest that they perform differential feature analyses of stimulus arrays, these explanations are not derived from any coherent theory of visual processing and thus remain speculations as to the perceptual basis of the R-I dimension.

Kilburg and Siegel (1973) have argued that the underlying basis for R-I differences is the process of visual feature analysis. They have argued that the Selfridge-Neisser model of pattern recognition (Neisser, 1966; Selfridge, 1959, "Pandemonium"; Selfridge \& Neisser, 1960) is theoretically useful in accounting for (and predicting) many of the performance differences between reflective and impulsive children. The Selfridge-Neisser model is hierarchical and is based on a program for letter recognition which emphasizes feature testing. The model assumes that there are several levels of mechanisms operating on incoming information: Level 1 mechanisms are stimulus samplers that get basic information into the system; Level 2 mechanisms are stimulus analyzers, each of which determines whether or 
not the stimulus has certain features. Results of these feature tests are conveyed to the next level, a set of "subroutines" which perform operations on the results of the feature tests. At the highest level, the probability values from these subroutines are compared and the item associated with the largest value is selected as the best "guess" as to the identity of the stimulus.

To test the hypothesis that reflective children perform a more detailed and thorough feature analysis (Level 2), Kilburg and Siegel (1973) tested reflective and impulsive first- and fifth-graders in a forced-choice recognition memory task. The possibility that correct recognition responses could be made on the basis of verbal labels, visual features, or both was systematically varied in the experimental conditions. Although reflective children made more correct recognition responses than did impulsive children under all experimental conditions, this difference was, as predicted, significant only in a condition in which the sole basis for a correct response was a visual feature analysis. Performance in this condition was equivalent to that in a condition in which a correct response could be made on the basis of either visual feature differences or the name of the stimulus; performance in these conditions was superior to the chance level performance in a condition in which the only basis for a correct response was the name of the stimulus. It was concluded that visual feature analysis independent of verbal processes was responsible for visual recognition memory.

Unfortunately, certain methodological problems in Kilburg and Siegel's (1973) study precluded adequate testing of the hypothesis. First, due to a problem with instructions, performance in a condition in which the correct and incorrect test stimuli differed in only one visual feature was not significantly better than chance. Second, in the condition in which a significant R.I difference was found, the number of feature differences between the correct and incorrect test stimuli was large ( 3 to 7 or 8 ) and varied unsystematically.

By systematically manipulating the number of feature differences between correct and incorrect test stimuli, the present study tests the hypothesis that reflective children engage in a more detailed visual feature analysis of a stimulus than do impulsive children. Reflective children should do better than impulsive children only when there are relatively few feature differences between correct and incorrect test stimuli. Moreover, since one criterion in deciding whether an individual is reflective or impulsive is response latency and since the Selfridge model can account for most of the choice reaction time data with adults (Smith, 1968), it was predicted that the expected R-I differences in correct responses would also be found in their response latencies.

\section{METHOD}

Subjects

Ninety-four white middle-class fifth-grade boys attending Pittsburgh Parochial Schools participated in the research (mean $\mathrm{CA}=10$ years 7 months).

\section{Stimuli}

The Matching Familiar Figures Test (Kagan et al, 1964) was used to classify Ss on the dimension of reflection-impulsivity (R-I). The MFF is a match-to-sample task in which $\mathrm{S}$ is shown a standard stimulus and is told to choose the one of eight variants that exactly matches the standard. The other seven variants differ from the standard in one small visual detail. Two practice and test items were given.

Stimuli for the recognition memory task consisted of a presentation deck and a test deck. The presentation deck consisted of $963 \times 5$ in. laminated cards on each of which was a black line drawing of a common object or animal. The test deck consisted of $965 \times 8$ in. laminated cards on each of which were two black line drawings.

\begin{abstract}
Apparatus
The apparatus consisted of a test stand on which each of the test cards was placed. At the bottom of the stand was a photocell-controlled microswitch wired to a Hunter timer (facing E) which started each time a new card was placed on the stand. In front of the stand and below the test card locus were two response buttons. Pressing either button automatically stopped the timer. E manually recorded the response latency (time between stimulus presentation and response selection).
\end{abstract}

\section{Procedure}

All 94 children were individually administered the MFF during a first session lasting approximately $25 \mathrm{~min}$. The essential instructions to the child were that he was always to point to the variant (on the lower page) that was exactly like the standard (on the upper page). For each of the 10 test items, E recorded the number of errors the child made (a maximum of six errors per item was allowed) and the response latency for each item (time from presentation to first response, whether correct or not). Children whose mean response latency was above the median $(13.01 \mathrm{sec})$ and whose total number of errors was below the median (25) were classified as reflective; children whose mean latency was below the median and whose total number of errors was above the median were classified as impulsive. Of the $94 \mathrm{Ss}$ tested, 32 were classified as reflective, 31 as impulsive. The 6 Ss whose scores fell at either median were excluded.

In a second session held approximately 2 weeks later, these children were individually administered the test of recognition memory by a different E. Each $S$ was seated, handed the presentation deck of 96 cards (same completely randomized order for all Ss), and given the following instructions: "Here is a deck of cards with drawings on them of animals and things that you have seen before. I want you to look carefully at each of the cards and go through the deck. When you finish looking at each card, turn it over and put it in the pile over there." Since children's recognition memory performance is depressed when stimulus presentation is paced by $\mathrm{E}$ (Kagan, personal communication), $\mathrm{S}$ was allowed to go through the deck at his own pace. Following this each $S$ was told: "Now, I'm going to show you more cards. Each one has two drawings on it. I'll put each card on the stand in front of you. I want you to look at both drawings and push the button underneath the one that you saw before in the first part of the game. Push the button as quickly as you can after you make your choice. If you are not sure which one it is, I want you to guess anyway." Each S was then shown all 96 test cards, one at a time. For each test card, E recorded whether the response was correct or incorrect and the latency of that response. Due to failure to understand the instructions or to stereotyped response choice, the data from three reflective and two impulsive children were not included.

\section{Experimental Conditions and Predictions}

The recognition test consisted of 96 cards, 24 for each of four experimental conditions. Within each deck of 24 , the correct figure was on the left for 12 of the cards and on the right for the other 12. Examples of presentation and recognition test 
De

Presentation Stimulus

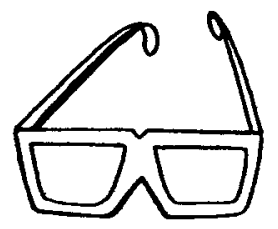

Recognition Test Item
2FD

Presentation Stimulus

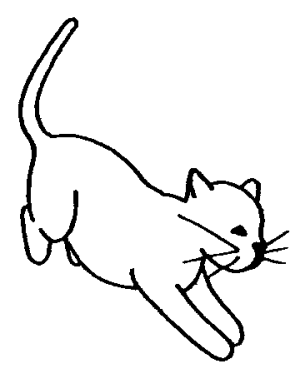

Reconition Test Itom
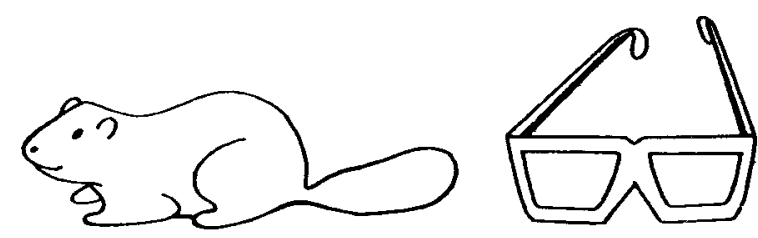

1FD

Presentation Stimulus

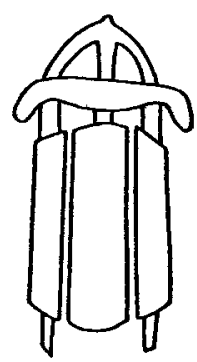

Recosnition Test Item

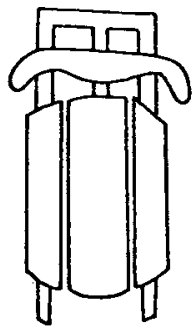

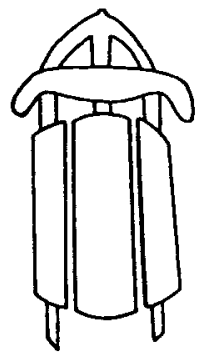
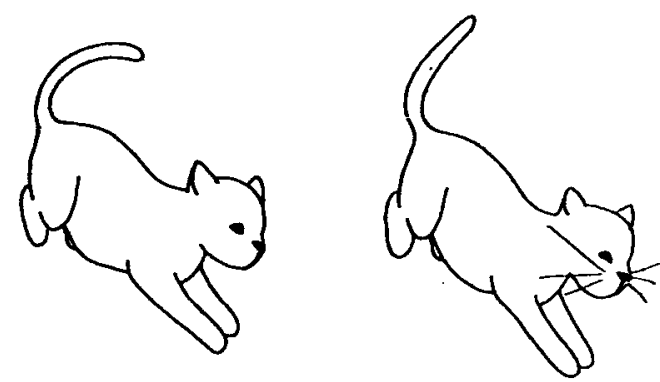

AFD

Presentation Stimulus

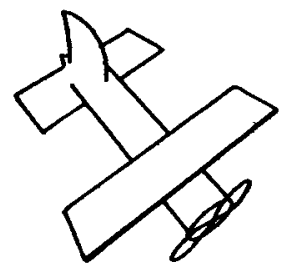

Recosnition Test Item

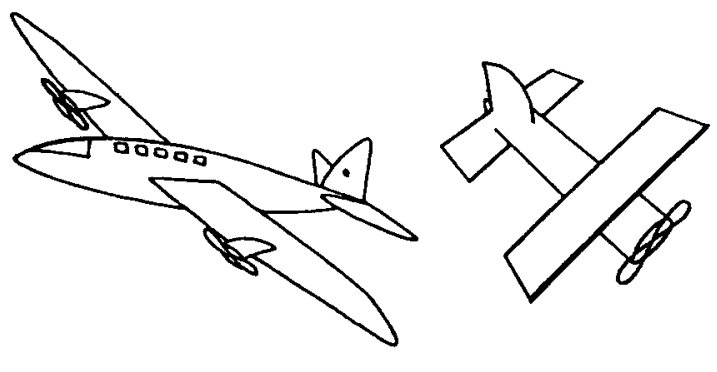

Fig. 1. Examples of presentation stimuli and recognition test items for each of the experimental conditions.

items for each of the four experimental conditions are presented in Fig. 1.

Condition DO (Different Object). Twenty-four stimuli were randomly chosen from the 96 original stimuli, and each was paired with a completely different object or animal on the test card (see Fig. 1). Since the correct and incorrect test stimuli had different names and also differed in an infinite number of visual features, this condition should produce a very high number of correct recognition responses. Since it was hypothesized that reflective and impulsive $S$ s differ primarily in their tendency to 
Table 1

Means and Standard Deviations for Reflective and Impulsive Ss: Number of Correct Responses and Latencies for Each Experimental Condition

\begin{tabular}{|c|c|c|c|c|c|c|c|c|c|}
\hline \multirow[b]{3}{*}{ Group } & \multirow[b]{3}{*}{$\mathrm{N}$} & \multicolumn{8}{|c|}{ Experimental Conditions } \\
\hline & & \multicolumn{2}{|c|}{ DO } & \multicolumn{2}{|c|}{$1 F D$} & \multicolumn{2}{|c|}{$2 \mathrm{FD}$} & \multicolumn{2}{|c|}{$4 \mathrm{FD}$} \\
\hline & & Mean & SD & Mean & SD & Mean & SD & Mean & SD \\
\hline $\begin{array}{c}\text { Correct Response } \\
\text { Reflective Ss } \\
\text { Impulsive Ss }\end{array}$ & $\begin{array}{l}29 \\
29\end{array}$ & $\begin{array}{l}22.59 \\
21.52\end{array}$ & $\begin{array}{l}1.57 \\
2.41\end{array}$ & $\begin{array}{l}18.28 \\
16.31\end{array}$ & $\begin{array}{l}1.94 \\
2.30\end{array}$ & $\begin{array}{l}18.24 \\
17.69\end{array}$ & $\begin{array}{l}2.17 \\
2.25\end{array}$ & $\begin{array}{l}22.72 \\
21.27\end{array}$ & $\begin{array}{l}1.19 \\
1.75\end{array}$ \\
\hline All Ss & 58 & 22.05 & 2.09 & 17.30 & 2.33 & 17.97 & 2.21 & 22.22 & 1.57 \\
\hline $\begin{array}{l}\text { Latencies } \\
\text { Reflective Ss } \\
\text { Impulsive Ss }\end{array}$ & $\begin{array}{l}29 \\
29\end{array}$ & $\begin{array}{l}2.46 \\
2.18\end{array}$ & $\begin{array}{l}1.14 \\
0.66\end{array}$ & $\begin{array}{l}3.15 \\
2.71\end{array}$ & $\begin{array}{l}1.34 \\
0.83\end{array}$ & $\begin{array}{l}2.74 \\
2.32\end{array}$ & $\begin{array}{l}0.96 \\
0.65\end{array}$ & $\begin{array}{l}2.26 \\
2.16\end{array}$ & $\begin{array}{l}0.79 \\
0.67\end{array}$ \\
\hline All Ss & 58 & 2.32 & 0.93 & 2.93 & 1.13 & 2.53 & 0.84 & 2.21 & 0.73 \\
\hline
\end{tabular}

perform detailed feature analyses and since a detailed visual feature analysis was not required to make a correct response to these stimuli, no performance difference between reflective and impulsive Ss was expected in this condition. Additionally, Kilburg and Siegel (1973) found no performance differences between reflective and impulsive Ss in an identical condition.

Condition IFD (One Feature Difference). Twenty-four different stimuli from the original presentation stimuli were each paired with another stimulus having the same name but differing from the original stimulus in one visual detail or feature (see Fig. 1).

Condition 2FD (Two Feature Differences). Twenty-four different stimuli from the original presentation stimuli were each paired with another stimulus having the same name but differing from the original stimulus in two visual details or features (see Fig. 1).

These two conditions should be the most difficult, since choosing the correct stimulus requires a rather complete visual feature analysis of the original stimulus during initial presentation. Performance in Condition 1FD should be poorer than that in $2 F D$, and performance in both should be poorer than in DO. But, in both Conditions 1FD and 2FD, reflective Ss should make more correct responses than impulsive Ss.

Condition 4FD (Four Feature Differences). The remaining 24 stimuli from the original presentation stimuli were each paired with another stimulus having the same name but differing from the original stimulus in four visual details or features (see Fig. 1). Kilburg and Siegel (1973) found that reflective Ss made more correct responses than impulsive $S$ s in a similar condition, but a large and variable number of features distinguished the correct from the incorrect test stimuli. Thus, it was not clear that the R-I difference would be significant when the number of feature differences was large (i.e., four) and constant. Performance in this condition should be better than that in $1 F D$ and $2 F D$. If recognition memory is determined by a process of visual feature analysis (independent of verbal labeling), then performance in Condition 4FD should be equivalent to that in Condition DO.

In short, reflective and impulsive Ss were predicted to differ in conditions where detailed visual feature analyses were required, but not in conditions where a more global feature analy sis would suffice to produce a correct response (i.e., DO and probably 4FD). Generally, it was expected that the greater the number of feature differences between correct and incorrect test stimuli, the better would be the recognition memory performance.

It was also expected that mean correct response latency would be related to the number of correct responses. Specifically, latencies should be longest in Condition $1 F D$, next longest in 2FD, and shortest in both DO and 4FD (the latter two should not differ). As with the data for correct responses, a R-I by Condition interaction was expected.

\section{Correct Responses}

\section{RESULTS}

The means and standard deviations of the number of correct responses made in each of the four experimental conditions by reflective and impulsive Ss are presented in Table 1. A 2 (R-I) by 29 (Ss) by 4 (conditions) repeated measures ANOVA was performed on the number of correct responses in each condition for each S. The main effect of R-I was significant $[F(1,56)=$ 9.04, $\mathrm{p}<.01]$ : Reflective Ss made significantly more total correct responses (81.83) than did impulsive Ss (77.24). As predicted, the main effect of condition was highly significant $[\mathrm{F}(3,168)=161.75, \mathrm{p}<.001]$. Scheffé $(.05)$ confidence intervals [MSE $=2.45$, critical value $(\mathrm{CV})=0.97]$ indicated that, as predicted, performance in Condition DO (22.05 correct responses) did not differ from that in 4FD (22.22), and performance in both DO and 4FD was significantly greater than that in both 2FD (17.97) and 1FD (17.30). Contrary to prediction, performance in $2 F D$ was not significantly greater than that in 1FD, but the difference was in the predicted direction. That performance in DO and 4FD was equivalent and that a correct response in 4FD could not be made on the basis of the name of the stimulus (e.g., both correct and incorrect test stimuli were airplanes) provides strong inferential evidence that visual recognition memory is determined by a process of visual feature analysis and that verbal labels have little or no direct effect on visual recognition performance.

The predicted R-I by Condition interaction was only marginally significant $[\mathrm{F}(3,168)=2.11, .05<\mathrm{p}<.10]$. Scheffé (.05) confidence intervals (MSE $=2.45, \mathrm{CV}=$ $1.57)$ indicated that reflective $S s$ made significantly more correct responses than impulsive Ss only in Condition IFD. This finding was crucial to, but only partially confirmed, the hypothesis (reflective and impulsive Ss did not differ in 2FD). As predicted, reflective and impulsive $S$ did not differ in Condition DO.

The differential performance of reflective and impulsive Ss on conditions in which correct and 
incorrect test stimuli were distinguished only by visual features was predicted from the Selfridge-Neisser feature-testing model. So, an additional 2 (R-I) by 29 (Ss) by 3 (conditions-1FD, 2FD, 4FD) repeated measures ANOVA was performed on the number of correct responses in each of these conditions for each $\mathrm{S}$. The main effect of R-I was significant $[F(1,56)=8.80$, $\mathrm{p}<.01]$ : Reflective Ss made more total correct responses in these three conditions (59.24) than did impulsive Ss (55.72). The main effect of condition was highly significant $[F(2,112)=170.31, p<.001]$ : Performance in Conditions $1 F D$ and 2FD was equivalent and significantly poorer than in 4FD. Most importantly, the R-I by Condition interaction was also significant $[F(2,112)=3.08, p<.05]$. Scheffé $(.05)$ confidence intervals $(\mathrm{MSE}=2.43, \mathrm{CV}=1.37)$ indicated that for reflective $\mathrm{Ss}$, performance in $1 \mathrm{FD}$ and $2 \mathrm{FD}$ was equivalent and poorer than in 4FD; for impulsive Ss, performance in $1 F D$ was poorer than in $2 F D$ and that in 2FD was poorer than in $4 F D$.

\section{Correct Response Latencies}

Each S's mean latency for each of the four experimental conditions was computed on the basis of correct responses only. The means and standard deviations of the correct response latencies in each of the four experimental conditions for reflective and impulsive Ss are also presented in Table 1. A 2 (R-I) by 29 (Ss) by 4 (conditions) repeated measures ANOVA was performed on the mean correct response latency in each condition for each $\mathrm{S}$. Only the main effect of condition was significant $[\mathrm{F}(3,168)=23.65, \mathrm{p}<.001]$. Scheffé (.05) confidence intervals (MSE $=.252, \mathrm{CV}=$ .26) indicated that the mean latency in Condition 1FD $(2.93 \mathrm{sec})$ was significantly longer than that in $2 \mathrm{FD}$ (2.53). Mean latencies in $1 \mathrm{FD}$ and $2 \mathrm{FD}$ were significantly longer than those in 4FD (2.21) and DO (2.32); those in 4FD and DO were equivalent. Neither the main effect of R-I $[F(1,56)=2.14]$ nor the R-I by Condition interaction $(F<1)$ was significant $(p>.10)$.

Since specific predictions about latencies had been derived from the Selfridge-Neisser model only for the "FD" conditions, an additional 2 (R-I) by 29 (Ss) by 3 (conditions-1FD, 2FD, 4FD) repeated measures ANOVA was performed on each S's mean correct response latency for each of these three conditions. As in the previous latency analysis, the main effect of R-I was not significant $[F(1,56)=2.13, p>.10]$, and the main effect of condition was highly significant $[F(2,112)=41.48, p<.001]$. Scheffé $(.05)$ confidence intervals (MSE $=.186, \mathrm{CV}=.20$ ) indicated that latency in Condition 1FD was significantly longer than in 2FD, and latency in $2 F D$ was significantly longer than in $4 F D$. The predicted R-I by Condition interaction was also significant $[F(2,112)=3.85, p<.05]$ and is presented graphically in Fig. 2. Scheffé (.05) confidence intervals (MSE $=.186, \mathrm{CV}=.38)$ indicated that the latencies of reflective $\mathrm{Ss}$ were significantly longer than those of

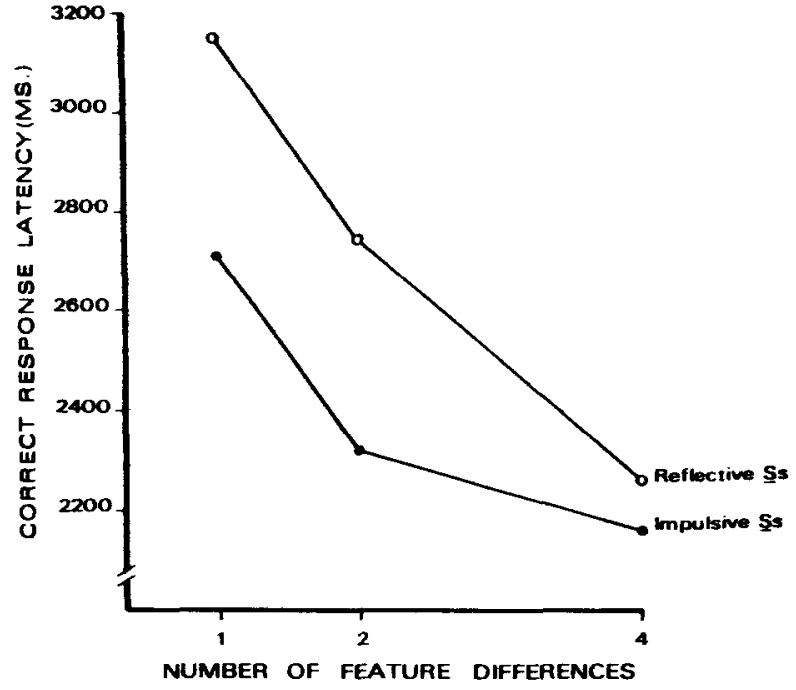

Fig. 2. Mean latency of correct responses of reflective and impulsive Ss in Conditions 1FD, 2FD, and 4FD.

impulsive Ss in Conditions $1 F D$ and $2 F D$, but not in 4FD. For reflective Ss, the mean latencies in all three conditions were significantly different from each other. For impulsive Ss, latency in $1 \mathrm{FD}$ was longer than that in 2FD and 4FD, but latency in $2 \mathrm{FD}$ was not significantly longer than in $4 F D$.

\section{DISCUSSION}

In general, the results of the present study are congruent with earlier research (e.g., Drake, 1970; Kilburg \& Siegel, 1973; Odom et al, 1971; Zelniker et al, 1972 ) in that performance differences between reflective and impulsive children were found on a task requiring visual feature analyses.

Although the performance of reflective Ss was superior to that of impulsive Ss in all conditions, this difference was significantly only in Condition 1FD. This finding supports the hypothesis that performance differences between reflective and impulsive children are greatest when a very detailed feature analysis is required. Moreover, since each of the MFF variants differ from the standard in only one visual feature, the finding of a R-I performance difference only in Condition 1FD takes on added significance. That is, these data argue that the underlying basis for the R-I dimension is a process of visual feature analysis rather than a broad cognitive disposition in tasks involving response uncertainty, and that reflective-impulsive performance differences can be specified by a feature-analytic model of pattern recognition.

That performance in Conditions 4FD and DO was equivalent further confirms our hypothesis. Although a correct recognition response could perhaps be made on the basis of the name of the stimulus in DO, a correct response in 4FD could not (i.e., both correct and incorrect test stimuli had the same names). Thus, strong 
inferential evidence is provided that correct recognition in both conditions is primarily dependent on visual processes and is relatively independent of verbal processes. That the pattern of correct response latencies paralleled that of correct responses further supports this contention.

Indeed, the response latency data from the FD conditions provide the strongest confirmation of the applicability of the Selfridge-Neisser model to R-I performance differences and recognition memory. Correct response latency was inversely related to the number of feature differences between correct and incorrect test stimuli (Fig. 2). The greater the number of feature differences between the correct and incorrect test stimuli, the shorter the mean response latency. That is, when there was only one feature difference between the correct and incorrect test stimuli, a very detailed feature analysis had to be performed during initial presentation, and a large number of feature tests had to be made during the test itself in order to make a correct response; the more tests that had to be performed, the longer the corresponding latency should be. That response latency in DO and $4 \mathrm{FD}$ were approximately equivalent is in line with the Selfridge-Neisser model, since the model allows feature tests to be carried out simultaneously. Our data indicate that this is the case when there are a large number of feature differences. As can be seen from Fig. 2, the time taken for several feature tests to be performed quickly reaches an asymptote at about four features.

Another finding that should not be overlooked concerns the efficiency of visual recognition memory. Even when the correct and incorrect test stimuli differ in only one distinctive feature, almost three-fourths of the stimuli were correctly recognized $(73 \%$ in Condition 1FD).

Our results suggest a caveat concerning the dimension of reflection-impulsivity: Since the relative effects of R-I on performance were weak relative to the effect of specific parametric values of the number of feature differences, it is highly likely that factors other than individual cognitive style differences (like R-I) account for the largest portion of the variance in recognition memory tasks. The magnitude of the R-I difference ( $8 \%$ in Condition 1FD) is hardly impressive and leads the present authors to question the fruitfulness of pursuing such a weak effect. Rather than trying to demonstrate R-I differences in a number of additional tasks, future research might well be directed towards investigating the specific conditions under which recognition memory might be enhanced for any child, reflective or impulsive. That the reflective and impulsive children did not differ in Condition 2FD might also indicate that the MFF (the instrument used to evaluate a child's position on the R-I dimension) should be rigorously reassessed. A variation in the number of feature differences between the variants and the standard might significantly affect the selection of reflective and impulsive $S s$ and the magnitude of performance differences between them.

In summary, the results from the present experiment indicate that (1) reflective and impulsive chi'dren differ in their propensity to engage in a detailed visual feature analysis; (2) visual feature analysis seems to be a most significant component in the underlying basis of the R-I dimension; (3) both the Selfridge-Neisser model and the present experimental paradigm are promising for future research in understanding the process of recognition memory.

\section{REFERENCES}

Drake, D. M. Perceptual correlates of impulsive and reflective behavior. Developmental Psychology, 1970, 2, 202-214.

Kagan, J. Reflection-impulsivity and reading ability in primary grade children. Child Development, 1965, 36, 609-628.

Kagan, J. Reflection-impulsivity: The generality and dynamics of conceptual tempo. Journal of Abnormal Psychology, 1966, 71, 17-24.

Kagan, J., \& Kogan, N. Individual variation in cognitive processes. In P. H. Mussen (Ed.), Carm ichael's manual of child psychology. Vol. 1. New York: Wiley, 1970. Pp. 1366-1378.

Kagan, J., Pearson, L., \& Welch, L. Conceptual impulsivity and inductive reasoning. Child Development, 1966, 37, 583-594.

Kagan, J., Rosman, B. L., Day, D., Albert, J., \& Phillips, W. Information processing in the child: Significance of analytic and reflective attitudes. Psychological Monographs, 1964, $78(1$, Whole No. 578).

Kilburg, R. R., \& Siegel, A. W. Differential feature analy sis in the recognition memory of reflective and impulsive children. Memory \& Cognition, 1973, 1, 413-419.

Neisser, U. Cognitive psychology. New York: Appleton-CenturyCrofts, 1967 .

Nuessle, W. Reflectivity as an influence on focussing behavior of children. Journal of Experimental Child Psychology, 1972, 14, 265-276.

Odom, R. D., McIntyre, C. W., \& Neale, G. S. The influence of cognitive style on perceptual learning. Child Development. $1971,42,883-892$.

Selfridge, O. G. Pandemonium: A paradigm for learning. In Mechanization of thought processes. London: Her Majesty's Stationery Office, 1959. Pp. 512-526.

Selfridge, O. G., \& Neisser, U. Pattern recognition by machine. Scientific American, 1960, 203, 60-68.

Smith, E. E. Choice reaction time: An analysis of the major theoretical positions. Psychological Bulletin, 1968, 69, $77-110$

Zelniker, T., Jeffrey, W. E., Ault, R., \& Parsons, J. Analy sis and modification of search strategies of impulsive and reflective children on the Matching Familiar Figures Test. Child Development, $1972,43,321-335$.

(Received for publication October 5, 1973; accepted October 21,1973 .) 